\title{
Encyclopedia
}

\section{Environmental Biophysics}

Created by: Jason Hubbart

Version received: 25 July 2019

Environmental Biophysics is the field of study of organisms and the physical environment (macro- to microenvironment) that they inhabit. In general, environmental biophysical research is designed to understand, a) the microclimate of a given organism of interest, b) how the organism functions (i.e. natural history) in its microenvironment, and c) how the organism responds to micro-environmental perturbation either caused by natural or anthropogenic processes. To become more attuned to the approach of environmental biophysics requires that one understand the primary components of the discipline including the fundamental concepts of microclimatology, environmental science, and biophysics. This entry includes descriptions of the basic components of environmental biophysics and importance of the field of study.

At no other time in history has the study of the environment and the relationship with the organisms that inhabit it been more important. Man's exploitations of the environment has resulted in a great deal of concern for the sustainability of natural resources, the rate of climate change, and the future of human continued population growth in the Earth's ecologically closed system. It is for this reason that there has never been a greater need to couple physically based principles and biological understanding to help human beings better steward the planet. Environmental biophysics integrates the broad fields of microclimatology, environmental sciences and biology (biophysics) to supply distinct quantitative characterization(s) of the requisite resources a given organism requires for survival.

A microenvironment can be thought of as a relatively small, localized area or microhabitat. In general, a microenvironment refers to a zone which has its own particular characteristics including (but not limited to) temperature, rainfall, wind exposure and topography. The combination of such physical characteristics creates a distinct and well defined micro-environment $[1]$. The most fundamental physical factor that influences microclimate is net solar radiation, which includes the absorption of solar radiation by day and the effective re-emission of long wave radiation, which occurs at all times but is most important in the absence of solar radiation at night. Micrometeorologists seek to understand the exchange processes of energy, mass and momentum between Earth's surface and the atmosphere[1][2].

It is the exchange processes between the atmosphere and biological organisms that forms the component of environmental biophysics with the greatest temporal and spatial variability. Exchange processes may include fluxes of water, heat, carbon and other bio-climatically relevant substances. Despite the often apparent direct dependence of these exchange processes on atmospheric gas exchanges, water vapor, and heat flux, scientists know relatively little about the mechanistic dynamics controlling them. For example, vegetation reacts to the state of the atmosphere, but also exacts control on local, regional and continental weather processes primarily through evaporation, transpiration and carbon assimilation. This is in part because terrestrial plant-atmosphere heat and gas exchange is heavily dependent on vegetative species composition, plant morphology, and vegetative canopy density [2]. Because these surface conditions can vary significantly over relatively small spatial scales, they can facilitate highly variable micro-scale patterns or interrelationships of atmospheric variables and surface cover types (e.g. vegetation). The same kinds of processes govern fauna life histories, though the relationships are even less well understood than for vegetation. This is largely due to the transient nature of most fauna and coupled complex metabolic pathways (e.g. endotherms). Regardless, understanding microclimate processes is critically important since related information can help to determine whether a given location is suitable to grow a forest, or soybeans, or to raise cattle, or create a wetland refuge for migrating geese $^{[2][3]}$.

Environmental processes are a critical component of environmental biophysics. Environmental science is conventionally an interdisciplinary field dealing with the scientific study of natural systems and human impacts on those systems. Most modern environmental problems are complex, combining both natural and anthropogenic perturbations, and demanding the problem-solving insights of multiple disciplines. Environmental scientists seek to understand natural processes (both 


\section{Encyclopedia}

physical and biological) and the role technology in human society and natural processes ${ }^{[3]}$. In terms of human environmental science, complex social demands must be understood and integrated with knowledge of technology and natural processes, including biophysical processes, to best manage human altered ecological systems sustainably.

Biophysics (or biological physics) is an interdisciplinary science that applies theories and methods of physical sciences to questions of biology, and focuses on disciplines including: bioenergetics, cell biophysics, channels, receptors and transporters, electrophysiology, membranes, muscle and contractility, nucleic acids, photobiophysics, proteins, supramolecular assemblies, spectroscopy, and many other areas ${ }^{[4]}$. Environmental biophysics is a broadly interdisciplinary science encapsulating the environmental, hydrological, ecological and biological sciences. Environmental biophysicists are interested in quantitatively characterizing the physical environment in which biological organisms reside, including interactions between organisms and the environment. However, they also focus on the flux of heat and mass transfer and models used to apply those fluxes between the organisms and their environment. Areas of study in environmental biophysics include: energy exchange, mass and momentum flux, the conservation of energy and mass, temperature, water and water vapor, wind, properties of gasses and liquids, conductance of heat and mass, soil heat flux, radiation and other foci[2][3][5]. Ultimately, the difference between biophysics and environmental biophysics could be argued to be primarily a matter of scale.

In a historical sense, given human beings interest in understanding themselves and their relationships to world in which they live, it is probable that the discipline of environmental biophysics began earlier than any other scientific discipline since being able to survive in one's environment has always been a matter of primary importance. However, the systematic application of the scientific method to environmental biophysical relationships goes back only for the previous few hundred years. The most recent advances in environmental biophysics have been tied to other disciplines such as hydrology with the implementation of mathematical models used to quantify energy and mass flux transfer, and the use of conservation principles. Notably, many theoretical models that have been created to describe energy and mass flux in hydrologic sciences can also be applied to processes between living organisms and their surroundings. The development of such models has in part been a result of improvements in sensing and data logging technology, allowing for data sets characterizing energy and mass fluxes at both high temporal and spatial scales, and thus creation and validation of highly quantitative physical processes based models.

Although a number of problems in environmental science deal with chemistry and social issues, physical aspects and the applications of environmental biophysics are increasingly important. For example, further development of sensors, the interaction of sunlight with airborne particulates and molecules, energy conservation processes, disposal/storage of radioactive wastes, development of energy saving and so-called "green" materials (e.g., polymers), and bio-physiological response mechanisms all contain interesting and challenging environmental biophysical implications [2][3][5][1]. In addition, the use of the physical sciences in developing technological innovations that modify lifestyles towards more environmentally benign directions is contributing to a better quality of life and thus quality biophysical environment. Integrating disciplines such as environmental science, microclimatology, and biophysics requires a thorough background and comprehensive training in mathematics and physics for critical problem solving and understanding of analytic tools in order to move the discipline of environmental biophysics progressively forward. However, when investigating environmental problems, physics can never be the sole method of resolution. Physics does however play a pivotal role in exploring, monitoring and above all understanding the world we live in, and our effects on it, both at a local and a global scale. Environmental biophysics provides the physical and biological framework to understand the interdisciplinary connectivity between physical processes and biological systems. To be an effective contemporary environmental biophysicist requires that one be familiar with the main concepts of biological sciences, ecological sciences, environmental science, and hydrologic science. The environmental biophysicists must then seek physiological, biochemical, and molecular connections and interactions. Therefore, by definition environmental biophysics is one of the most interdisciplinary and integrated sciences, and may hold some of the greatest future discoveries of any scientific discipline.

\section{References}

1. Robson, R.E., and Blake, D.. Physical Principles of Meteorology and Environmental Physics: Global, Synoptic and Micro Scales; World Scientific Publishing Co. Pte. Ltd.: 5 Toh Tuck Link, Singapore 596224, 2008; pp. 288.

2. Bonan, G. Ecological Climatology: Concepts and Applications-Third Edition; Cambridge Press: United Kingdom, 2016 ; pp. 754. 
3. Campbell, G.S., and Norman, J.M.. An Introduction to Environmental Biophysics-Second Edition; Springer-Verlag: New York, USA, 1998; pp. 286.

4. Glaser, R. Biophysics: An Introduction-Second Edition; Springer Heidelberg : Dordrecht London New York, $2012 ;$ pp. 407.

5. Monteith, J.L., and Unsworth, M.H.. Principles of Environmental Physics, Plants, Animals, and the Atmosphere-Fourth Edition; Academic Press: London, 2013; pp. 395.

\section{Keywords}

Environmental Science; Microclimatology; Ecological Climatology; Ecology; Biology; Physical Hydrology; Radiation; Biophysics; Plant Ecology; Physics 